\title{
QUALITY OF LIFE IN PATIENTS WITH POLYRADICULONEURITIS, WHO WERE STATIONATED IN RIGA EAST UNIVERSITY HOSPITAL "GAILsEZERS"
}

\author{
Alisa Rjabikova1,4, Ulla Bambīte'1, 5, Illa Mihejeva²,3, Aivars Vētra² \\ 1Riga Stradinš University, Faculty of Continuing Education \\ 2Riga Stradinš University, Department of Rehabilitation, Riga East University hospital \\ 3 Riga East University hospital \\ 4 Children's Clinical University Hospital \\ ${ }^{5}$ North Kurzeme Regional Hospital
}

Introduction: Polyradiculoneuritis - is a rare condition, associated with inflammatory reactions in nerves and nerve roots, and cause inability, which can lead to disability. According to National Institute of Neurological Disease and Stroke, the incidence of Guillain-Barre syndrome (the most common cause of polyradiculoneuritis) is 1-2:100,000 people per year (Guillain-Barré Syndrome Fact Sheet, NINDS, July 2011). There are no specific data in Latvia about the prevalence of polyradiculoneuritis and functional state of patients after leaving the hospital.

Purpose: Identify the main complaints, functional disorders, life quality changes patients suffer from polyradiculoneuritis one year after leaving the hospital.

Method: Qualitative research includes Riga East University Hospital Gailezers patients from 18 years old, with ICD-10 diagnoses G61-G63, for time period 01.01.2015-31.12.2016. Medical cards, evaluation protocols from electronic database Ärsta birojs, telephone survey, using 20-Item Short Form Health Survey, translated to Latvian, were used. Data processed with Excel and SPSS 19.0 programs.
Results: 46 patients were hospitalized (24 males, 22 females) with G61-G63 diagnoses, one death was recorded due to illness complications on the 7th hospitalization day. Median age - 59 years $(S D=17.07)$, hospitalization duration -15.48 days $(S D=$ 7.63). Interviewed 17 respondents: 2 claimed their current state of health as excellent (11.76\%), $5(29.41 \%)$ - good, 6 (35.29\%) medium, $5(24.41 \%)$ - as bad. Physically challenging activities can't perform $12(70.59 \%)$; daily activities are difficult for 6 $(35.29 \%), 7(41,18 \%)$ feel pain, $8(47,06 \%)$ have sensory disorders. Social activities limited in 6 (35.29\%), 13 (76.47\%) felt stress last month due to complications after polyradiculoneuritis.

Discussion and Conclusion: Patients with polyradiculoneuritis continue to experience functional impairment associated with movement, daily activities, pain, and emotional state one year after discharging from the hospital. A systematic approach and follow up is needed to evaluate the patients with polyradiculoneuritis.

\section{Patients' functional statement, exiting from the hospital}

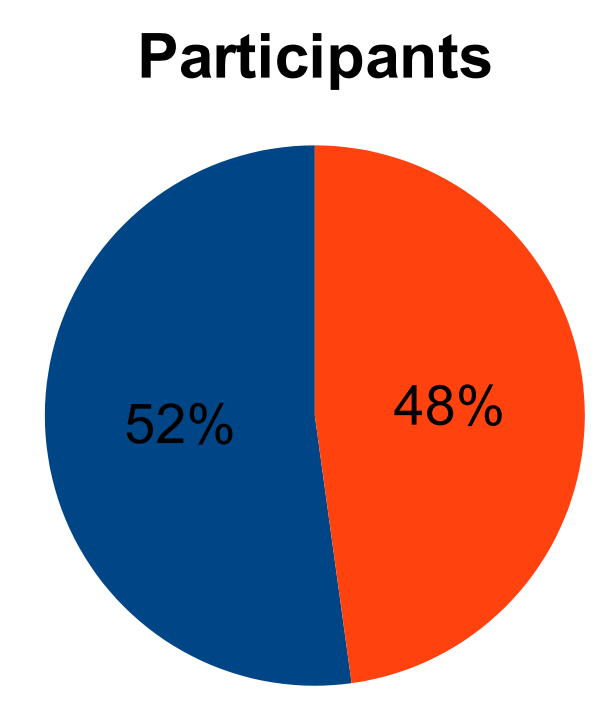

- Male 24 Female 22

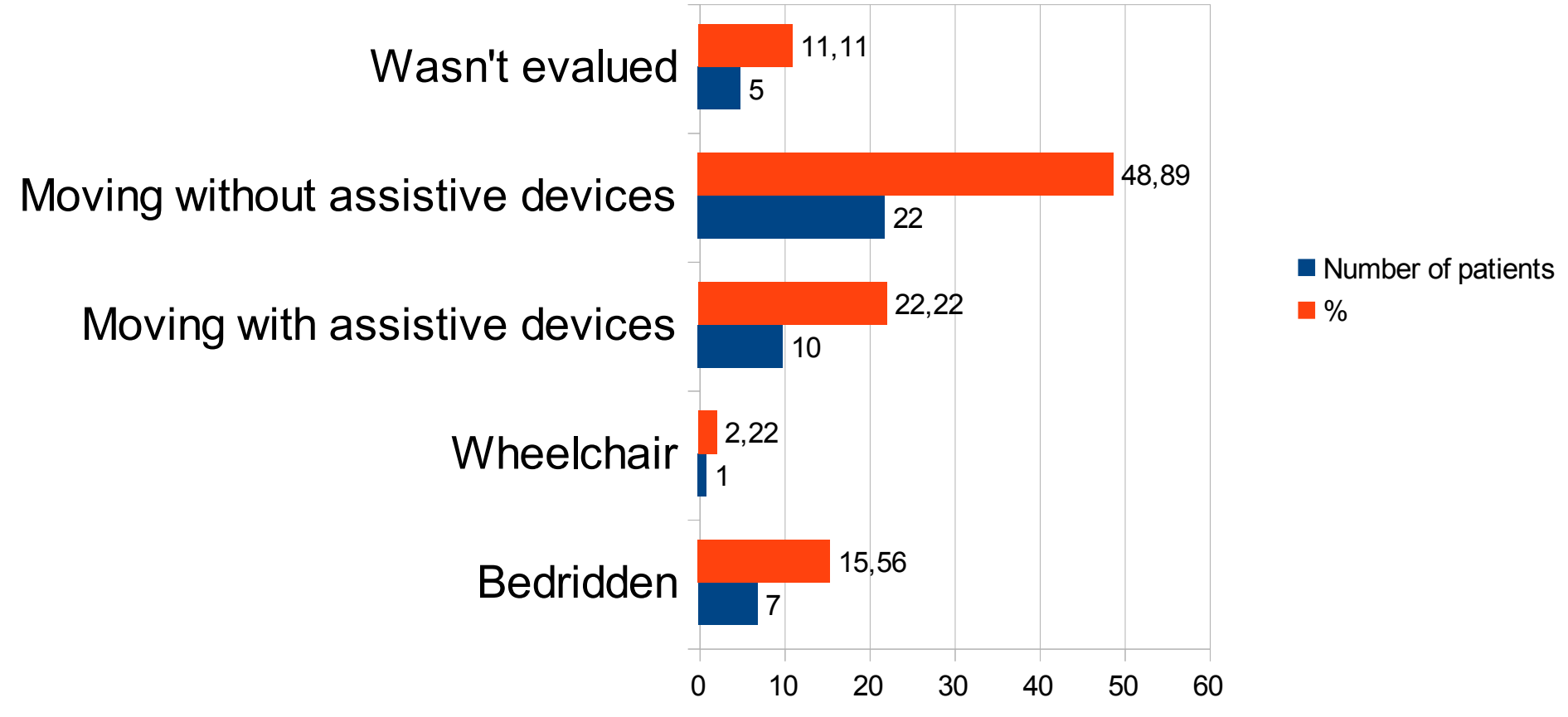

Functional disorders, according to SF-20 (20-Item Short Form Health Survey)

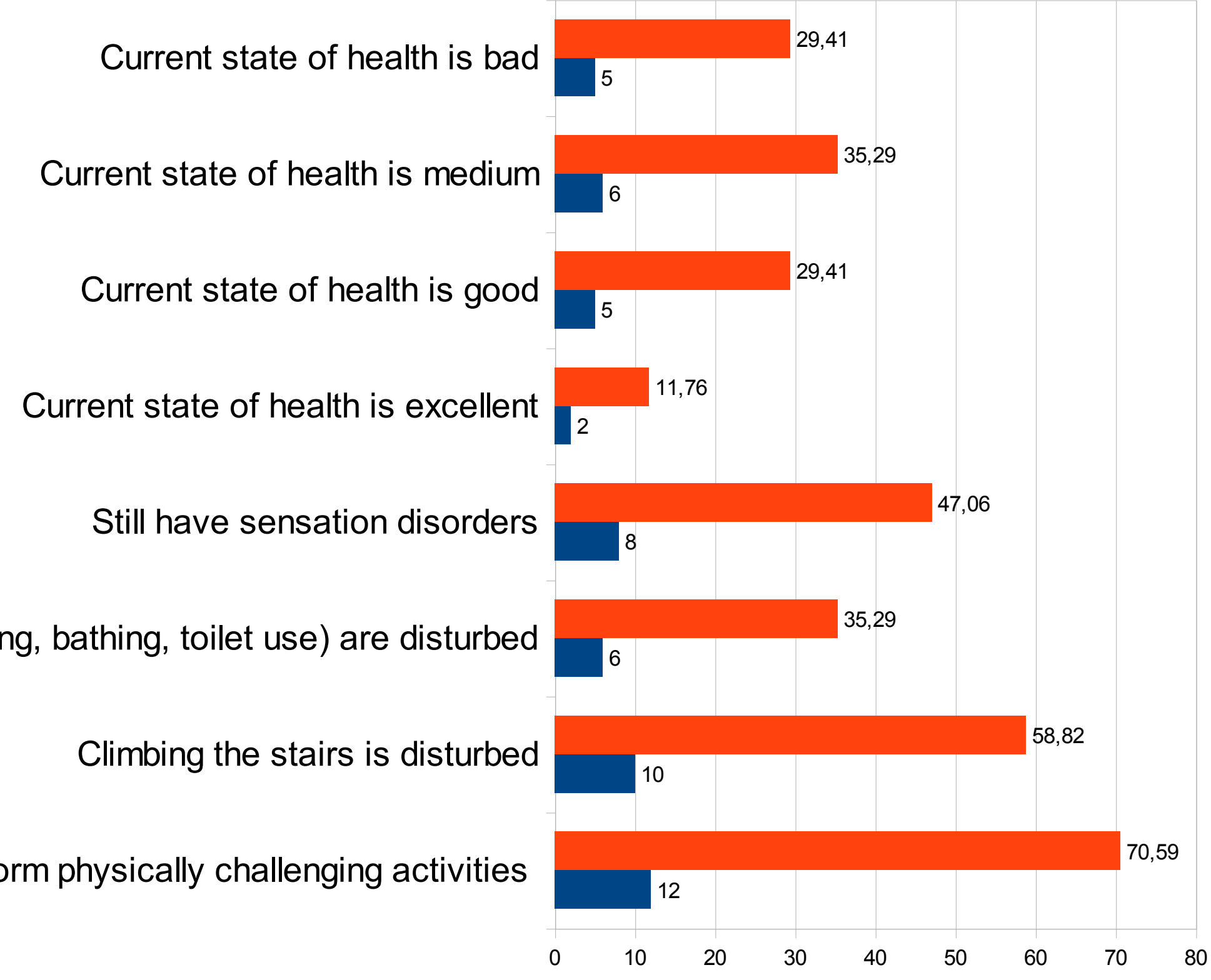

